This, he says, may be due to the absorbing power of the casein molecules, which hold back the larger portion of it. If the enzymes are united to some albumin not passing through the filter, this union may account for their absence in the serum.

It is more probable, however, that they are separate bodies, having properties similar to those of the albumin, and of a closely allied nature. They remain active until the coagulation temperature of the lactalbumin is reached, $72-80^{\circ}$, and this is perhaps their own coagulation temperature.

Chrcago, Ilininots.

[CONTRIBUTION FROM THE ChEMICAI LABORATORY OF THE UNIVERSITY OF ILIINOIS.]

\title{
ORGANIC CHEMICAL REAGENTS. I. DIMETHYLGLYOXIME.
} By Roger Adams and Oliver KaMM,

Received June 20, 1918.

The manufacture of less common organic chemicals at the University of Illinois ${ }^{1}$ has reached the stage where much time is involved merely in preparing large amounts of certain substances, whose method of production has been thoroughly worked out. This part of the work is now occupying the full time of several chemists. The work at Illinois was taken up for two purposes: first, to supply chemicals which were entirely out of the market, and second, to give graduate students experience, partly in developing ordinary laboratory processes to large scale manufacture which might be directly transferred to plant production, and partly in devising new processes capable of development on a commercial scale. The second purpose is defeated as soon as the men must devote too large a portion of their energy to the routine manufacture of certain compounds. This difficulty could be avoided, however, if one or more chemical concerns would be willing to produce the most needed of these sub. stances. Undoubtedly, the reason this has not yet been done is because of the scarcity of research chemists and their preoccupation with larger and more important problems.

In view of the above situation it has seemed advisable to publish full details of the preparation of several of the more useful organic chemical reagents which are being made at Illinois so that they may be available to any manufacturer, thus allowing him to produce these substances quickly and without the expensive research work which is ordinarily necessary. The materials will continue to be made at the University until such a time as the commercial concerns are able to put them on the market at a reasonable price. It is hoped that the publication of these preparations may also serve the second purpose of helping out the scientific institution which may prefer for one reason or another to make their own reagents.

1 J. Ind. \& Eng. Chem., 9, 685(1917); This Journal, 40, 869 (1918); Science, 57, No. 1210, 225(1918). 
Particular care has been taken in the directions given below and will be taken in future publications to give full enough details so that a comparatively inexperienced chemist, if careful, can get good results on the first trial. So many preparations are described in text-books and in organic chemical literature which read well, but which in reality require. a large amount of study before they can be successfully repeated. An attempt will also be made, wherever possible, to insert what will happen provided the details described are not followed strictly. In this article, directions for dimethylglyoxime are given; but later the directions for other reagents such as methylene iodide, malonic ester, amylene, etc., will appear. Only those will be published in the chemical journals, however, for which there is considerable demand and in the preparation of which distinct advances have been made over what is now available in the literature. In the coming fall it is the intention to issue a University of Illinois pamphlet to be sold at cost which will include not only these directions, but also those for many other varied preparations which have been studied and developed at Illinois.

\section{Dimethylglyoxime.}

Dimethylglyoxime is now undoubtedly the most important reagent for the quantitative determination of nickel. This substance has been made by several methods, ${ }^{1}$ but the only one adaptable easily and cheaply to large scale production, is that by Gandarin. ${ }^{2}$. This consists in treating methylethylketone with amyl nitrite and hydrochloric acid to give the nitrosomethylethylketone. In alkaline solution this substance rearranges to the monoxime of diacetyl, which by heating with hydroxylamine goes readily to dimethylglyoxime. The reactions run smoothly. and the product is always obtained, although generally in smaller yields and sometimes in much smaller yields than are mentioned in the literature. The chief difficulty which arose in this country when dimethylglyoxime became scarce was that the necessary hydroxylamine salts were still scarcer and the preparation of them proved to be a stumbling block not easily overcome.

Gandarin's method has been studied thoroughly. Besides making many useful and important minor observations regarding his process two distinct improvements have been found. The first is that a crude solution of hydroxylamine, sulfate is perfectly satisfactory to use in place of a pure hydroxylamine salt. The details for making this crude solution have been developed. The second is the discovery of a good method of purification of the dimethylglyoxime when it is colored. The ma-

${ }^{1}$ Ber., I6, I80 (1883); Bl., [3] 6, 830 (1891); J.prakt. Chem., [2] 51, 550 (I895); Ann., 249, 204 (1888); 288, 27 (1895); Ber., 38, 2520 (1905); Z. anorg. Chem., 46, 144 (1905); Z. angev. Chem., I9, 1793 (1900).

I. prakt. Chem., [2] 77, 414 (1908). 
terial obtained is seldom white and the colored impurity can be removed only with great difficulty or not at all by crystallization. Gandarin suggested acidifying the sodium hydroxide solution of the colored product with acetic acid, but the results from this method are far from satisfactory. It has been found that the sodium hydroxide solution of the colored dimethylglyoxime when treated with excess of ammonium chloride solution yields colorless dimethylglyoxime, the impurity remaining in solution. The credit for this method of purification is due to Mr. V. Voorhees.

The development of the procedure will be divided into 7 parts:

r. Preparation of hydroxylamine sulfate.

2. Preparation of amyl nitrite.

3. Methylethyl ketone.

4. Preparation of nitrosomethylethylketone.

5. Preparation of dimethylglyoxime.

6. Purification of colored dimethylglyoxime.

7. Recrystallization of dimethylglyoxime.

\section{Hydroxylamine Sulfate.}

From among the various methods for the preparation of hydroxylamine salts, only two seemed feasible to attempt on a large scale. The electrolytic reduction of nitric acid in the presence of hydrochloric acid as carried out by Schoch ${ }^{1}$ proved unsatisfactory for the manufacture of large quantities of material as compared with the reduction of sodium nitrite with sulfur dioxide. The latter method was suggested by Raschez, ${ }^{2}$ and perfected by Divers. ${ }^{3}$ The details for carrying out the process are given below:

Four liters of distilled water are run into a 5 -liter round bottom flask fitted with a good stirrer and surrounded (stoneware jar) by a freezing mixture of ice and salt ( 5 parts ice to I part of sodium chloride). $325 \mathrm{~g}$. of soda ash and $420 \mathrm{~g}$. of technical sodium nitrite are then added. After the temperature has dropped to $-5^{\circ}$, a stream of sulfur dioxide is run into the solution at such a rate that the temperature is always kept below $0^{\circ}$ throughout the reduction. This temperature control is very important and low yields may be traced to the fact that the temperature has gone above $0^{\circ}$. If efficient cooling is provided the reduction is complete after 3 or 4 hours; this is indicated by the fact that the solution becomes acid in reaction towards litmus. Just before the solution is acid, it becomes slightly brown and gives off a characteristic oxide of nitrogen odor. The hydroxylamine is now present as the disulfonic acid.

1 This Journal, 38,12042 (1916).

2 Ann., 241, 161 (1882).

'J. Chem. Soc., 69, 1665 (1896). 
For the preparation of about $2 \mathrm{ko}$. of hydroxylamine sulfate at one time, a battery of 6 flasks is used. The stirrers are all run by one small electric motor. The supply of sulfur dioxide is obtained directly from an iron tank connected in parallel with the flasks. The glass tubes leading into the flasks are provided with individual glass stopcocks so that the rate of flow of sulfur dioxide into each flask may be regulated as desired. Practically no gas escapes except after the reduction is completed and hence the operation need not be carried on in a hood.

The liquid is siphoned from the flasks, placed in a 4-gallon earthenware jar (5-liter flasks and then subsequent partial evaporation in large porcelain dishes may be used), and heated in a boiling water bath for 48 hours, care being taken that the solution is distinctly acid before heating. If it is not acid, a small quantity of sulfuric acid must be added, otherwise much decomposition takes place during evaporation and the yields are very small. The solution should be allowed to evaporate during the twoday heating period, and it has generally reached, at the end of this time, a concentration of about one-half of the original volume, a point where further evaporation is unnecessary. If such is not the case, the solution should be heated still longer on the steam bath till it has reached about the volume mentioned above. The hot solution is now neutralized with sodium carbonate, methyl orange being used as an indicator. It has been found advantageous to add no excess of the carbonate, in fact, it is advisable to keep the solution slightly acid, otherwise free hydroxylamine is produced and considerable decomposition will take place. After neutralization, the solution is allowed to stand overnight at room temperature, during which time considerable sodium sulfate crystallizes. Hydroxylamine sulfate is very soluble in water, and will, therefore, remain in solution. It is advisable, however, to filter off the sodium sulfate and wash it twice with small portions of cold water. These washings are best worked up with the next run of hydroxylamine.

The filtrate from the sodium sulfate should contain about one-fourth gram of hydroxylamine sulfate per cc. of solution. If the solution is more dilute than expected, it is acidified with sulfuric acid, concentrated on a steam bath, neutralized with sodium carbonate, cooled to crystallize out any portion of sodium sulfate and again filtered. The amount of hydroxylamine salt present is determined by titrating about $2 \mathrm{cc}$. of the solution with an approximately $2 \%$ potassium permanganate solution which has previously been standardized with a pure sample of hydroxylamine sulfate. The titration is carried out in acid solution. More concentrated hydroxylamine sulfate solution may undoubtedly be used, but indications pointed to a poorer yield of the hydroxylamine sulfate when evaporation to much greater concentration took place.

The $25 \%$ solution of hydroxylamine sulfate as prepared above still 
contains a considerable amount of sodium sulfate together with ammonium sulfate. The solution, however, may be used directly for the preparation of dimethylglyoxime or other oximes. The yield of hydroxylamine sulfate from $2.5 \mathrm{ko}$. of sodium nitrite averages slightly above $2 \mathrm{ko}$. The time required by one student for the preparation of 2 ko. of hydroxylamine sulfate averages 6 hours, provided the preparation is run continously. One to two hundred pounds of ice are used.

Notes.-Attention is again directed to the necessity of maintaining the sodium nitrite mixture at a low temperature (slightly below $0^{\circ}$ ) throughout the reduction period. Low yields may invariably be traced to poor temperature control.

Hydroxylamine solutions attack the skin, and any workman who is preparing this material continuously should be provided with rubber gloves.

Calcium carbonate may be used for neutralization in place of sodium carbonate but its use offers no special advantage.

\section{Amyl Nitrite.}

I $200 \mathrm{cc}$. of amyl alcohol (technical grade, b. p. I28-132 $2^{\circ}$, is satisfactory) are placed in a 5 -liter copper can and $1000 \mathrm{~g}$. of technical sodium nitrite are added. The mixture is cooled to $10^{\circ}$ by surrounding it with ice in a 4-gal. stoneware jar. A copper stirrer of the wing type run by a motor is used to stir the mixture of alcohol and nitrite. $300 \mathrm{cc}$. of conc. sulfuric acid are now added very slowly from a dropping funnel. During this procedure, the nitrite must be kept from settling to the bottom. This is easily accomplished by so vigorous stirring that a clear layer of alcohol is never visible above the nitrite. While the acid is running in, the temperature is an important consideration and should always be kept below $25^{\circ}$. The first $100 \mathrm{cc}$. of acid may be added rather rapidly, as the temperature rises only slightly, the second Ioo cc. must be run in more slowly, the third Ioo cc. very slowly and the thermometer watched closely as the reaction is likely to be violent towards the end. If the temperature goes much above $25^{\circ}$ large amounts of oxides of nitrogen are evolved and lost.

When all of the acid has been added, the stirring is continued until no more brown fumes appear above the liquid. The copper can is then removed from the ice bath and water added. The nitrite which rises to the surface is poured off into a large separatory funnel and more water is added to the salt mixture. The latter is stirred well, allowed to settle, and the amyl nitrite again poured off, together with most of the water. The extraction is continued until no more nitrite rises to the surface. It is not necessary to dissolve all of the salt, but the small lumps must be broken up, and the remaining salt should be perfectly white.

The nitrite is washed once with clean water to remove any acid, dried 
with fused calcium chloride and distilled. The fraction boiling at $92^{-}$ $100^{\circ}$ is used in subsequent experiments. A yield of $\mathrm{r} 300 \mathrm{cc}$. is obtained.

Notes.-'Two runs are easily made at the same time, although it is advisable to use individual motors.

The reaction proceeds very smoothly when $C$. P. sodium nitrite is used, whereas the technical product tends to produce a rather violent reaction after about $200 \mathrm{cc}$. of acid have been added. It is, therefore, advisable to add the acid very cautiously and to use efficient metal stirrers to break up the salt cake. The above yields are based upon technical sodium nitrite.

Glass apparatus may be used in place of copper cans, but it is much more difficult to control the temperature and consequently the yields are poorer.

\section{Methylethylketone.}

Methylethylketone is obtained as a by-product in the commercial distillation of acetone and may be bought on the market. The technical product is of fair purity and usually requires no further fractionation. Since it invariably contains water which interferes with the reaction involved, the ketone should be dried for one day over about 10\% of its weight of calcium chloride. It is then transferred to a fresh portion of calcium chloride, where it remains until used. The calcium chloride used for the final drying of one portion of ketone is used for the first drying of the next portion. In this manner, loss of ketone due to absorption by the drying agent is partly avoided!

\section{Nitrosomethylethylketone.}

A 5 -liter flask is fitted with a stirrer, a thermometer, and a $300 \mathrm{cc}$. dropping funnel. The flask is adjusted at such a height that a pan for warming or cooling the solution may be raised or lowered under the flask as needed. $850 \mathrm{cc}$. of dry methylethylketone is poured into the flask and the stirrer started. $30 \mathrm{cc}$. of conc. hydrochloric acid is added and the liquid is warmed to $40^{\circ}$, during which procedure the color of the liquid usually becomes dark reddish brown. The warm water is siphoned from the pan, and ice and cold water added. I $200 \mathrm{cc}$. of amyl nitrite are then added slowly, keeping the temperature between $40-50^{\circ}$. Heat is generated as the nitrite is added and cooling is required. The nitrite is best run in $250 \mathrm{cc}$. portions, each portion being added during about 30 minutes and the total amount certainly in not less than two hours. The solution is stirred vigorously during the entire time. A slight evaporation of the ketone undoubtedly takes place during this operation, so that on a larger scale it would probably be advisable to have a reflux condenser. After all of the nitrite has been added the temperature should be $50^{\circ}$. The cooling mixture is then removed but the stirring is continued for $\mathrm{I}_{5}$ to 30 minutes longer. 
The reaction mixture now consists of a solution of nitrosomethylethylketone in amyl alcohol. The ketone must be extracted with sodium hydroxide solution and the amyl alcohol recovered to be reconverted into amyl nitrite. The mixture is therefore treated with a cold solution of $45 \circ \mathrm{g}$. of sodium hydroxide dissolved in one liter of water. Meanwhile the stirrer is worked rapidly enough so that no distinct layer of alcohol is present. The stirring is continued for 30 minutes. The light yellow solution assumes a reddish brown color upon the addition of alkali.

The reaction mixture is now transferred to a large separatory funnel and the amyl alcohol layer separated. In order to insure a complete removal of amyl alcohol, the alkaline solution is shaken with one or sometimes two portions of $300 \mathrm{cc}$. of ether. (Undoubtedly other less expensive solvents, like benzene, could be used for this purpose.) From these extracts ether and amyl alcohol are recovered. By fractionation about $75 \%$ of the original amyl alcohol, boiling $120-132^{\circ}$, is obtained and used again for amyl nitrite. The low boiling fraction consists of amyl alcohol, methylethylketone, and a little amyl nitrite.

\section{Dimethylglyoxime.}

The solution of the sodium salt of nitrosomethylethylketone is divided into two equal parts. Each fraction is poured into a 5-liter flask and the amount of hydroxylamine sulfate solution equivalent to $400 \mathrm{~g}$. of hydroxylamine sulfate is added to each portion. Before the hydroxylamine sulfate is added, it is made neutral to litmus by the addition of sodium hydroxide. The volume of the entire solution in each flask should not exceed 3.5 liters. The flasks are immediately placed on the steam bath and are allowed to remain for about one hour with frequent shaking.

Some oxime usually precipitates out as soon as the hydroxylamine is added and rises to the surface, but the larger portion appears after heating, as a yellow fluffy solid. Ammonia is liberated when the solution is heated and this may cause the oxime to froth on shaking. The precipitated oxime changes in color from brown to light yellow, during the heating.

After heating for not more than one hour, the oxime is immediately filtered off while the solution is still hot and washed with hot water. If left on the water bath for a longer period or allowed to cool before filtering, it will turn brown and will not wash white. The mother liquors are poured back into the flasks and cooled rapidly. A concentrated solution of ammonium chloride is added in order to precipitate any dimethylglyoxime that remains in solution as the sodium salt. This second precipitate is filtered off on a separate filter and washed with hot water. Usually two liters of wash water are used for each portion. The oxime is allowed to remain on the suction filter for 20 to 30 minutes and is then spread out on filter paper to dry. The yield of product from a double 
portion ( 1700 cc. ketone), after being dried at room temperature for 3 days, weighs IIOO to 1200 grams. If the directions have been followed carefully the product will be practically white and will possess, even before crystallization, the correct melting point. It is even pure enough to use directly for quantitative determinations, but on account of small amounts of mechanical impurities, it is best to recrystallize it from alcohol. If the directions have not been carefully followed, the product will be colored from light pink to a medium shade of brown. The colored products usually cannot be obtained perfectly white by crystallization, and must be subjected to the special purification method given below, in order to remove the color. As a matter of fact, the colored material recrystallized gives perfectly satisfactory results in nickel analyses, but is not good enough in appearance for a high grade commercial product.

\section{Purification of Colored Dimethylglyoxime.}

Any colored dimethylglyoxime, together with the final residues from the alcoholic solutions used for recrystallization, may be purified in the following manner: The product is dissolved in an $8 \%$ sodium hydroxide solution to saturation, which is accomplished on the steam bath with occasional shaking. The dark solution is filtered and to the hot filtrate is added a concentrated solution of ammonium chloride in excess of the amount required to precipitate all of the dimethylglyoxime. The product is filtered off at once, using suction, and washed well with boiling water. If not fairly white at this point, it is well to repeat the sodium hydroxide solution and precipitation.

The dried precipitate is washed and recrystallized from alcohol as directed below. The filtrates from the precipitation process are rejected with the little dimethylglyoxime they hold in solution, care being taken to assure the absence of the sodium salt, by the addition of ammonium chloride.

\section{Crystallization of Dimethylglyoxime.}

A preliminary washing removes much of the color from any slightly impure product, but usually this treatment will be found unnecessary if the crude material is almost white. If the washing seems advisable, about one ko. of crude dimethylglyoxime is moistened with about twice its weight of $95 \%$ ethyl alcohol. The mixture is heated to boiling on the water bath, and after being well stirred is filtered hot with suction. The product on the filter is washed with a small amount of cold alcohol. The wash alcohol is, of course, reserved for washing one or more other portions of material and is finally distilled and the materials recovered.

For the crystallization of the washed material or of the crude material itself, the usual procedure for crystallization is followed. About 200 to $25^{\circ} \mathrm{g}$. of the product is placed in a 5 -liter flask together with about 3 liters of $95 \%$ alcohol and the mixture heated to the boiling point on the 
water bath. After a saturated alcoholic solution of dimethylglyoxime is obtained the solution is filtered with suction through a heated funnel. It is usually advisable to place a cloth filter beneath the usual filter paper. In this manner, clogging of the filter is avoided.

The filtrate is transferred to a clean 5 -liter flask and cooled rapidly with frequent shaking so as to produce small crystals. This is advisable since dimethylglyoxime is used in alcoholic solution and the large crystals, because of their slow rate of solution, are not desired. The crop of white crystals is filtered off, washed with cold alcohol, and the filtrate transferred to the first flask, which still contains some of the material from the first run. An additional portion of crude dimethylglyoxime is added and the operations repeated.

The alcoholic filtrates are used repeatedly until they become rather brown in color, when they are distilled to recover not only the alcohol but also the colored dimethylglyoxime residues which may be purified by conversion into the sodium salt and reprecipitation with ammonium chloride as already directed above.

In filtering the hot alcohol solutions the vacuum should be so controlled that no large amount of solvent is lost due to volatilization.

Time Required for the Preparation of Dimethylglyoxime.

Following the above directions, two students working 8 hours a day are easily enabled to prepare $1000 \mathrm{~g}$. of recrystallized dimethylglyoxime per day. In actual practice one individual prepares the hydroxylamine solution, and also recrystallizes the final dimethylglyoxime. Two ko. of hydroxylamine sulfate (in solution) are prepared in one day, whereas $1600 \mathrm{~g}$. are actually needed in the preparation.

The other individual prepares the $2400 \mathrm{cc}$. of amyl nitrite required, from which, with two portions of methylethylketone of $850 \mathrm{cc}$. each, he prepares the nitroso derivative. The nitrosomethylethylketone thus obtained is run in 4 portions with hydroxylamine sulfate ( $400 \mathrm{~g}$. for each portion) to the final product- $1200 \mathrm{~g}$. of uncrystallized dimethylglyoxime. The yield of recrystallized product is $1000 \mathrm{~g}$. The material is white and crystalline, and melts very sharply at $240^{\circ}$ (corr.).

\section{NEW BOOK.}

Oude Chemische Werktuigen en Laboratoria Van Zosimus tot Boerhaave. By DR. H. J. BACKER. University of Groningen. 62 pages, 52 illustrations. J. B. Wolters U. M. Groningen and the Hague, I918.

This attractive pamphlet follows the lines of an address delivered by the author at the opening exercises of the new organic laboratory of the University of Groningen in I9I7.

A brief introduction dealing with the available sources of information 